\title{
Nanogap fabrication by joule heating of electromechanically spun suspended carbon nanofibers
}

\author{
Arnoldo Salazar, ${ }^{\mathrm{a}, \dagger}$ Braulio Cardenas-Benitez, ${ }^{\mathrm{b}, \dagger}$ Bidhan Pramanick, ${ }^{\mathrm{b}}$ Marc J. Madou,,${ }^{\mathrm{a},}$ and \\ Sergio O. Martinez-Chapa,
}

${ }^{a}$ Department of Mechanical and Aerospace Engineering, University of California, Irvine, 92697 CA, USA

${ }^{b}$ School of Engineering and Sciences, Tecnologico de Monterrey, Av. Eugenio Garza Sada 2501 Sur, 64849 Monterrey, NL, Mexico

$\dagger$ Arnoldo Salazar and Braulio Cardenas-Benitez contributed equally to this work.

*Corresponding author. Tel.: (949)-824-6585. E-mail: mmadou@ uci.edu (Marc J. Madou). *Corresponding author. Tel.: +52 818-328-4095. E-mail: smart@ itesm.mx (Sergio O. MartinezChapa). 


\begin{abstract}
We present Suspended Carbon Nanofibers featuring a central nanometric gap fabricated by integration of Electro-Mechanical Spinning, pyrolysis of ultraviolet-cured microstructures and a Joule heating process. Photopatterned walls were used to suspend polymeric electrospun fibers based on a solution of SU-8. After pyrolysis, the complete structure was converted into a monolithic carbon microstructure featuring stable ohmic contact between the suspended fibers and the supporting walls without the need of further processing. By applying an electrical bias through the electrodes, the wire can be gradually thinned by excessive Joule heating, eventually forming a nanogap. The maximum supported current density in the fibers was found to be of the order of $10^{5} \mathrm{~A} / \mathrm{cm}^{2}$. Furthermore, the electrical characteristics of carbon nanofibers and the dimensions of the nanogaps were demonstrated to be dependent on the length of the fiber and atmosphere conditions, i.e. air or vacuum. A finite element simulation was used to gain insight into the influence of length on nanogap size. The shorter fibers modeled portrayed a significantly steeper temperature gradient, which agrees with the experimental observation of smaller gaps. The presented method facilitates an inexpensive manufacturing technique of nanogaps as small as $12 \mathrm{~nm}$, holding potential for the preparation molecular electronics devices.
\end{abstract}

\title{
1. Introduction
}

Electrodes separated by a nanometric distance, known as nanogaps, have been extensively applied in the fundamental study of the electrical properties and manipulation of sub-micrometric particles and molecules [1-4]. To fulfill both goals, a nanogap device must have a separation distance comparable to the size of the manipulated nanoscale object [5]. Sub-20 nm nanogap devices have been proposed by matching the gap size with the length of target DNA chains to 
study the electric conductivity of single bases [6]. In the case of particle manipulation, the application of a voltage between the tips of a nanogap can generate very high electric fields that, in turn, lead to the trapping of the target object through dielectrophoretic forces [2]. This principle has been applied to the active and nondestructive assembly of long DNA fragments ( 27 nm) [6], nanoparticles $(20 \mathrm{~nm})$ [7], and even micron-long carbon nanotubes (CNTs) [8]. In view of these applications, extensive research has been conducted in nanogap fabrication and several methods have been proposed, including focused ion beam (FIB) [9], mechanical break junction [10-12], electron beam lithography (EBL) [13], electron-beam induced oxidation [14], electrochemical synthesis [15], oblique angle deposition [16], amongst others. Unfortunately, these techniques still face serious technical challenges and suffer from low throughput, poor scalability, complex processing protocols and/or high cost of equipment [13]. Indeed, few methods offer the ability to routinely produce sub-50 $\mathrm{nm}$ nanogaps [17], especially when integrating them into microelectromechanical systems (MEMS).

Nanogap fabrication can also be achieved by electrical breakdown of metallic nanowires, usually predefined by two-dimensional photolithographic techniques like electron beam lithography (EBL) [4]. While allowing for controlled positioning and integration of individual wire components, EBL is not well suited for the preparation of multiple devices and leads to structures confined to a flat substrate [18]. An important alternative to metal-interconnects are one-dimensional carbon nanotubes (CNTs) and carbon nanofibers (CNFs), which can be used to produce nanogaps by electrical field-induced breakdown to create freely-standing gaps $[4,19]$.

In both of these one-dimensional carbon structures, the mechanism for nanogap formation under the application of a high current density is not based on atomic drift, but rather due to excessive Joule heating, which induces the breaking [20,21]. Carbon-based devices offer several 
advantages over metal electrodes, including better contacts to organic molecules due to strong CC bonding [22], biocompatibility [4], excellent electrical and thermal conductivities, and even semiconductor-like properties which prevent quenching of the excited states in electricallytriggered luminescent molecular junctions [19]. Cutting CNTs with electric currents usually yields nanogap electrodes with sub-10 $\mathrm{nm}$ separations, which are then functionalized to covalently attach the target molecule to create the molecular device $[19,23,24]$.

Nanogap platforms are highly dependent on the structural configuration of the gap electrodes and the substrate. In the case of CNFs and CNTs in particular, it is desirable that their position and orientation be controlled within a circuit, and that they be connected to metallic electrodes with robust and reproducible ohmic contacts [8]. Nonetheless, the traditional fabrication schemes for CNTs, such as chemical vapor deposition (CVD) fail to achieve this, as nanowires must be synthesized, isolated and placed on a flat substrate, followed by deposition of top-contact metal electrodes $[20,21,25]$. A limiting factor in the performance of such nanostructures is the high contact resistance at the interface between the carbon and the metal pads commonly used to connect to them. Therefore, an alternative approach to the fabrication of carbon nanowires is based on far field electrospinning (FFES) [26], where electrified jets of polymer solutions are used to produce nanofibers, followed by pyrolysis to carbonize the deposited polymer wires. Sharma et al. [26] reported the fabrication of suspended polymer nanowires over photopatterned walls. They subjected the suspended polymer fibers and supporting walls to pyrolysis in an inert $\left(\mathrm{N}_{2}\right)$ environment, obtaining monolithic carbon structures. The contact between the suspended carbon nanowires and the supporting walls is ohmic, as it avoids non-linear contact resistance effects that limit the performance of the resulting devices. An added advantage of suspending the nanostructure is that deleterious substrate influences such as contamination and charge shunting 
can be avoided [27], which are factors that critically affect conductivity measurements in DNAnanogap junctions, for example [6,9].

Due to the inherent bending instabilities of the liquid jet in conventional far field electrospinning (FFES), the positioning of single electrospun fibers is difficult to control [41,42]. In near-field electrospinning (NFES) one accomplishes better fiber deposition control by reducing the applied voltage and the distance between the dispensing needle and the grounded substrate [43]. Unfortunately, voltage reduction in NFES prevents electrospun fibers from stretching to the nanometric scale. In 2011, Bisht et al. [44] reported on the use of a new electrospinning technique known as Electro-Mechanical Spinning (EMS), which improved upon NFES by reducing the applied voltage and nozzle-to-substrate distance even further. In EMS, by mechanically pulling on the fiber and optimizing the viscoelastic properties of the polymer solution, one is able obtain thinner polymer fibers. To fabricate polymer nanowires through EMS that can also be carbonized successfully, Canton et al. [45] formulated a SU-8/polyethylene oxide $(\mathrm{PEO}) /$ tetrabutylammonium tetrafluoroborate polymer solution that was compatible with EMS, because of its sufficiently high viscoelasticity (through the high molecular weight of PEO) and its high carbon content (due to the carbon rich SU-8). This optimized ink formulation was used to deposit nanofibers on top of photolithographically patterned electrodes. The resulting structure was subsequently pyrolyzed, yielding a monolithic suspended carbon nanofiber structure with good ohmic contacts between the electrodes and the fibers. The obtained electromechanically spun carbon fibers had diameters ranging from a few micrometers, down to $200 \mathrm{~nm}[46]$.

In this paper, we describe a relatively simple and inexpensive fabrication method of nanogaps based on Joule Heating of suspended CNFs produced by EMS. The resulting carbon devices 
present stable ohmic contacts between wire electrodes and supporting walls without the need of further process steps. We confirmed this by obtaining the linear current-voltage relation of the wall-fiber-wall structure at lower biases. At higher biases, CNFs show considerable Joule heating, leading to nonlinear current-voltage behavior, gradual fiber thinning, and eventual fiber breakdown in the middle area of the wire. By conducting the breakdown experiments in a vacuum, the effects of oxidation and adsorption of other gases in air were minimized. Both the supported current density before breakdown, of the order of $10^{5} \mathrm{~A} / \mathrm{cm}^{2}$, and the resulting nanogap size were found to be dependent on fiber length, consistent with previous studies on heat diffusion in CNFs $[32,47,48]$. Computer simulation was employed to analyze the temperature distribution that arises when stimulating the suspended nanostructures by ohmic heating; we found that the steepness of the temperature profile is closely related to the portion of the wire that undergoes sublimation. Using the presented manufacturing method, we were able to routinely obtain sub-50 nm carbon nanogaps, and a minimum nanogap size of $12 \mathrm{~nm}$.

\section{Experimental}

\subsection{Carbon Nanofiber Arrays}

The fabrication of the suspended nanofiber devices comprised three main steps. First, UV photolithography was used to pattern SU-8 2025 (MicroChem Inc., MA, USA) supporting walls ranging in thickness from 2 to $30 \mu \mathrm{m}$ and separation from 3 to $50 \mu \mathrm{m}$. Second, a polymer fiber was deposited over the walls created in the previous step by EMS, as described in earlier publications $[44,45]$. In order to obtain multiple fibers with similar composition, length $(L)$ and diameter $(D)$, six supporting walls were fabricated per device, and during the EMS step a single polymer fiber was deposited (Fig. 1a). In the third step, the complete nanofiber/SU-8 wall 
compound was converted into glassy carbon by pyrolysis at $900^{\circ} \mathrm{C}$ in an inert environment; this process resulted in a monolithic carbon structure with five suspended carbon fibers of equal $L$ and $D$ (Fig. 1b and Fig. 1c). By effect of the carbonization step, the ultraviolet-cured resins were thermally decomposed at high temperatures, resulting in the loss of non-carbon atoms and volumetric shrinkage [45]. The upper portion of the support walls shrank considerably more than the lower part, which was firmly anchored to the substrate, causing an increase in the trench size at the upper part and resulting in elongation of the fiber.

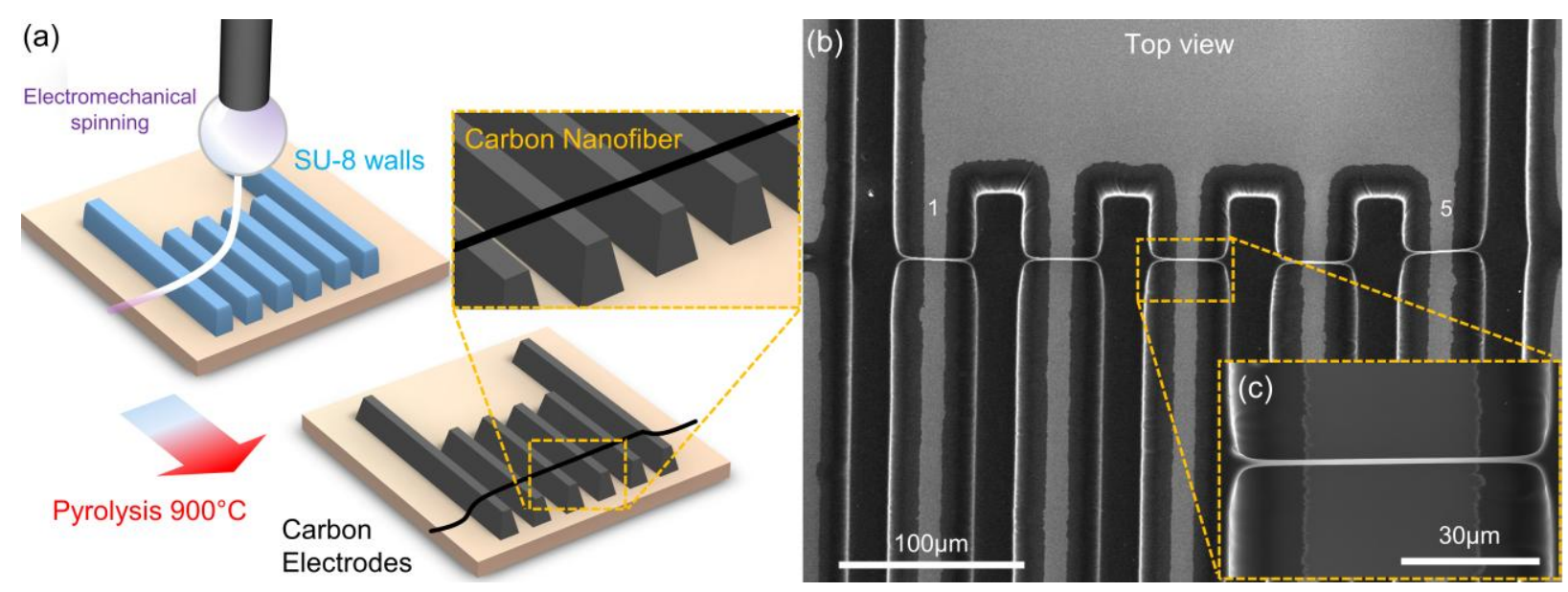

Fig. 1. (a) Overview of the fabrication scheme used to produce CNF arrays, combining ElectroMechanical Spinning and carbonization of the structures by pyrolysis. (b) Scanning electron micrograph of the six-wall structure containing five CNFs (top view). (c) Close- up of an individual suspended CNF bridging two carbon electrodes.

Using this method, we fabricated a total of fifty-three CNFs ranging in $L$ from 5 to $65 \mu \mathrm{m}$ and $D$ from 233 to $2200 \mathrm{~nm}$. Furthermore, the six-wall structure facilitated the sequential electrical probing of the fibers and is easily scalable for the simultaneous manufacturing of large numbers of CNFs. 


\subsection{Fabrication of Nanogaps}

From the obtained CNFs arrays, forty samples (eight devices, each with 5 fibers) were electrically biased until breakdown to fabricate nanoscale gaps. Electrical characterization and breakdown experiments were performed using a Keithley 2636B source meter and a Kleindiek probe system. The electrical breakdown of the CNFs was performed in two steps. In the first step, a voltage ramp starting from $0 \mathrm{~V}$ was applied individually to each CNF. As the voltage was gradually increased, samples started experiencing noticeable heating, which was evidenced by a decrease in resistance. The second step consisted of stopping the ramping at that voltage and changing the stimulation to constant bias. Because the temperature increase due to Joule heating was significantly high at that point, fibers experienced thinning and eventual breakdown. This failure revealed itself as a sudden increase in resistance (open-circuit), leaving behind the nanoscale gap. Furthermore, the effect of oxidation on the nanowire breaking mechanism was observed by conducting experiments in a vacuum of $8 \times 10^{-6}$ Torr and in air. Measurement of the dimensions of the fiber and the nanogaps were made with a FEI Magellan 400 XHR SEM and a FEI Quanta 3D FEG Dual Beam SEM.

\subsection{Computational modeling of Joule heating}

To calculate the heat transferred to CNFs by electrical current application, a computational model was built with COMSOL Multiphysics $5.0^{\circ}$. The fibers and carbon electrodes were identified as different domains. The fiber geometry consisted of a cylinder with a diameter of 1 $\mu \mathrm{m}$, with five different lengths $(5,10,20,40$ and $65 \mu \mathrm{m})$. The two parallel electrodes contacting the fiber were rectangular blocks $(10 \mu \mathrm{m}$ thick, $40 \mu \mathrm{m}$ wide and $120 \mu \mathrm{m}$ long) intended to approximate the geometries presented in Fig. 1b and Fig. 1c. An electrical conductivity of 100 
$\mathrm{S} / \mathrm{cm}$ was used for such structures [49], whereas for carbon fibers, a value of $300 \mathrm{~S} / \mathrm{cm}$ was selected based on average conductivity values observed in our experiments. Thermal conductivities of glassy carbon [50] $(6.4 \mathrm{~W} / \mathrm{m} \cdot \mathrm{K})$ and CNFs [51] $(\sim 12 \mathrm{~W} / \mathrm{m} \cdot \mathrm{K})$ were used for the electrode and the wire domains, respectively. A diffuse surface boundary condition was set at all the exposed surfaces in the geometry, with an emissivity value of 0.8 [52]. The only surfaces unexposed to the ambient were the faces of the electrodes in contact with the substrate, which were set at a temperature of $293.15 \mathrm{~K}$ for all simulation purposes.

\section{Results and discussion}

\subsection{Electrical probing of carbon nanofibers}

The electrical properties of the CNFs were analyzed with a two-probe I-V method, rather than a four-probe technique. Contact resistance and spreading resistance can be neglected in the devices, as fibers are integrated to the carbon walls with ohmic contacts and their resistance is much greater compared to the carbon electrodes, as a result of their size and geometry difference [26,45,53]. Fig. 2a shows typical I-V curves of three suspended CNFs, all with $L=44 \mu \mathrm{m}$, but with different diameters and different maximum applied voltages $\left(V_{\max }\right)$. As expected, thinner fibers have larger overall resistance and therefore need smaller current to induce breaking by Joule heating. Moreover, at the low voltage end of the I-V curve in Fig. 2a, a linear relation between current and voltage is observed, but as heating effects begin to take over with increasing voltage, the resistance of CNFs decreases and the relationship becomes nonlinear. At this point in the experiment, stimulation was changed to constant-voltage, as it is close to the electric current threshold value that induces breakdown of the CNF. Indeed, it was found that thicker samples could sustain higher voltages for longer periods of time, while thinner ones experienced 
a more sudden breaking. During the constant voltage phase of the test, two competing temperature-related mechanisms affect the resistance (Fig. 2b): i) the temperature-dependent conductivity in glassy carbon nanowire structures [38,53,54,], which causes a reduction in resistance as the temperature in the $\mathrm{CNF}$ increases; ii) towards the end of the fiber's lifetime, a steep increase in the resistance is observed due to the gradual reduction in its cross-section after reaching a critical temperature value that is high enough to sublimate carbon $[55,56]$.
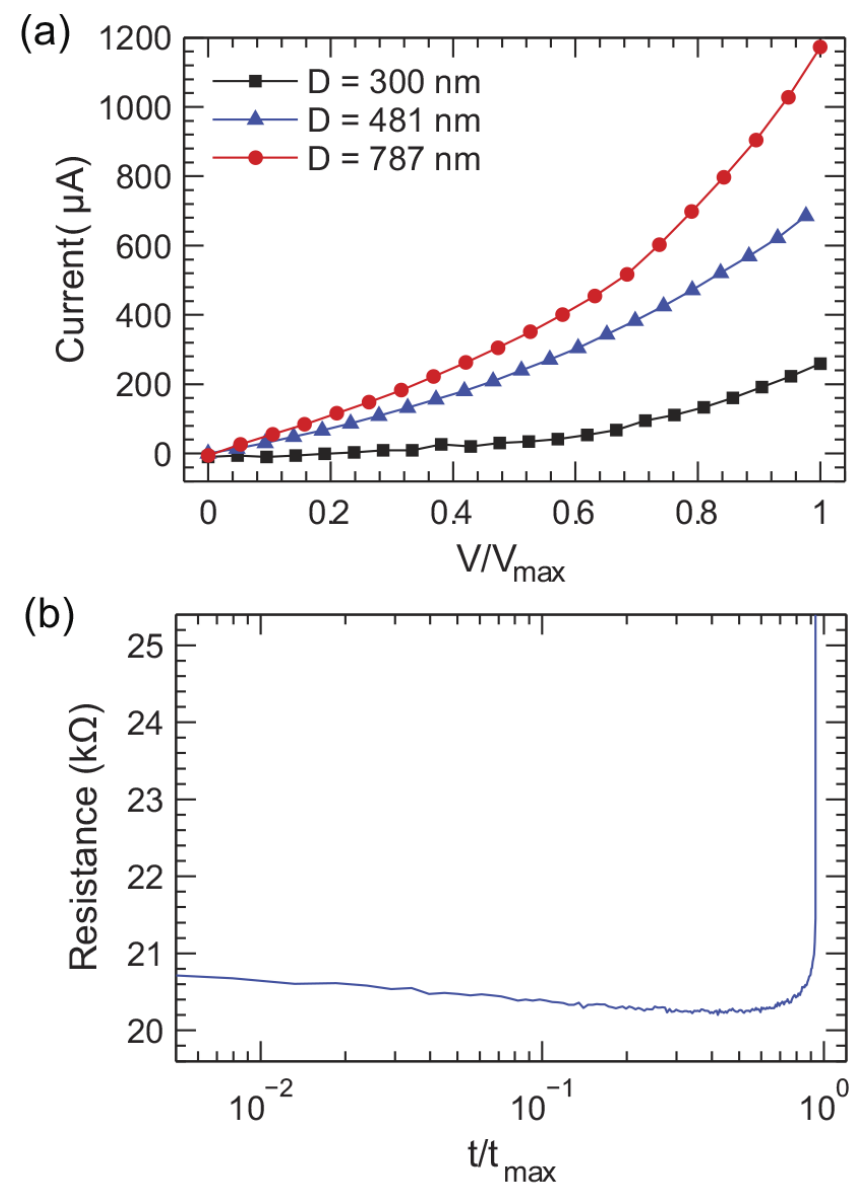

Fig. 2. (a) I-V characteristics of three fibers of same length $(L=44 \mu \mathrm{m})$ and different $D$. $V_{\max }=3.4$ $\mathrm{V}, 5.2 \mathrm{~V}, 3.0 \mathrm{~V}$ and $t_{\mathrm{max}}=0.021 \mathrm{~s}, 0.043 \mathrm{~s}$ and $0.38 \mathrm{~s}$ for CNFs with $D=300 \mathrm{~nm}, 481 \mathrm{~nm}$ and 787 $\mathrm{nm}$, respectively. (b) Resistance evolution with time for a fiber of $L=44 \mu \mathrm{m}$ and $D=300 \mathrm{~nm}$. 
Following the procedure outlined above, the fifty-three fibers (ranging in diameter from $233 \mathrm{~nm}$ to $2200 \mathrm{~nm}$ ) were stimulated with constant voltages in the range of 2 to $6 \mathrm{~V}$, and the current density at the point of their breakdown $\left(J_{M}\right)$ was registered. While no clear trend was observed for the current density as a function of $D$, an inversely proportional relation to $L$ was identified (Fig. 3a), similar to the results previously reported for CNFs grown by plasma-enhanced CVD $[31,32]$, and consistent with diffusive heat transport in one-dimensional conductors. From current testing experiments, it was concluded that the breakdown current density for CNFs is of the order of $\sim 10^{5} \mathrm{~A} / \mathrm{cm}^{2}$, which is consistent with the $J_{M}$ values found on carbon nanowires characterized as amorphous in nature [28]. These values are several orders of magnitude lower than the reported values for CNTs $\left(\sim 10^{8} \mathrm{~A} / \mathrm{cm}^{2}\right)$ [29]. Such deviation from CNTs is expected, as photoresist-derived CNFs have a highly glassy carbon composition, rather than a graphitic structure [30]. Despite the low current sustainability recorded for CNFs, it is important to note that the $J_{M}$ value for a carbon nanowire structure is dependent on the $L$ of the conductor channel [31]; in general, shorter carbon nanowire samples will exhibit higher $J_{M}$ values due to the Joule heating effects on electrical breakdown [32].This behavior can be verified in Fig. 3a, where the shortest samples $(L=5.0 \mu \mathrm{m}$, located at the rightmost part of the plot) reach values of up to $10^{6} \mathrm{~A} / \mathrm{cm}^{2}$. These values of $J_{M}$ are close to those found in CNFs produced by other techniques, such as plasma-enhanced CVD, which place $J_{M}$ around $1 \times 10^{6} \mathrm{~A} / \mathrm{cm}^{2}$ to $5 \times 10^{6} \mathrm{~A} / \mathrm{cm}^{2}$ [31].

In glassy carbon materials, electric properties are a result of the $\mathrm{sp}^{2} / \mathrm{sp}^{3}$ ratio and crystallinity present in their microstructure [33]. Because the CNFs presented in this work are derived from carbonization of a photoresist precursor at relatively low pyrolysis temperatures $\left(900^{\circ} \mathrm{C}\right)$, their composition is expected to be glassy in nature [34]. Hence, to gain insight into the microstructure of the CNFs produced by EMS, we measured the Raman spectra of samples with different 
diameters. Fig. $3 \mathrm{~b}$ shows the Raman spectrum of a CNF with $D=467 \mathrm{~nm}$, where the wide Dband near $1350 \mathrm{~cm}^{-1}$ is characteristic of disordered carbon, while the G-band is reveals the $\mathrm{sp}^{2}$ content of the sample. In addition, the peak intensity ratio of the bands $\left(I_{D} / I_{G}\right)$ presented in Fig. $3 b$ is greater than unity, indicating a predominantly disordered carbon microstructure [35]. Furthermore, Fig. 3c presents the relationship between the measured peak intensity ratio $I_{D} /$ $I_{G}$ and $D$ for $20 \mathrm{CNF}$ samples. In this graph, data points are distributed between $1<I_{D} / I_{G}<$ 1.7, showing no clear dependence on $D$. Other works on ES of CNFs have linked their graphitic content to the alignment of polymer chains during electrospinning, prior to carbonization [26]. Nonetheless, the absence of a clear trend between $D$ and $I_{D} / I_{G}$ suggests that the ES parameters here used did not have a significant effect on the molecular alignment of the precursor molecules. The Raman spectroscopy characterization is consistent with the results found for $J_{M}$ that suggest a low graphitic content in the samples. Moreover, the I-V curve non-linearity presented in Fig. 2a can be explained by the fact that electric transport in disordered carbons like glassy carbon is generally caused by thermally-activated, hopping conduction [37-40]. Therefore, it is concluded from the Raman spectroscopy and electric characterization that the CNFs here presented have a predominantly glassy carbon microstructure that limits their current carrying capacity.

(a)

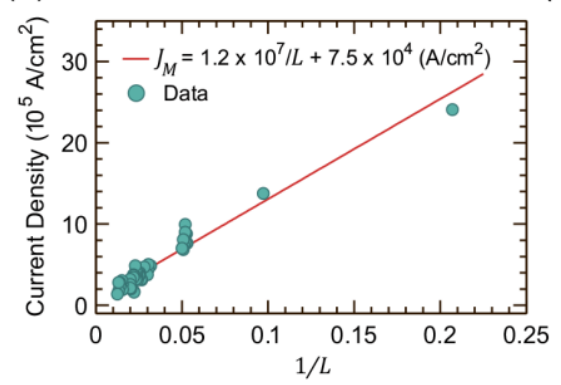

(b)

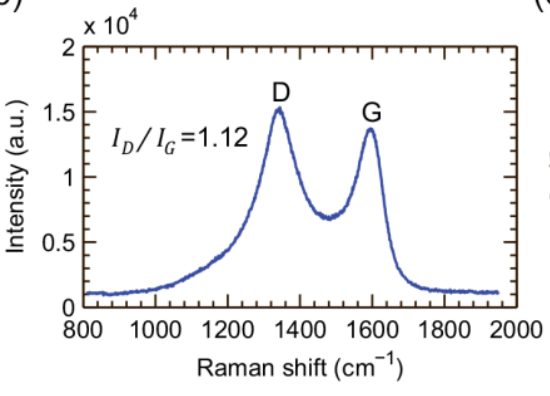

(c)

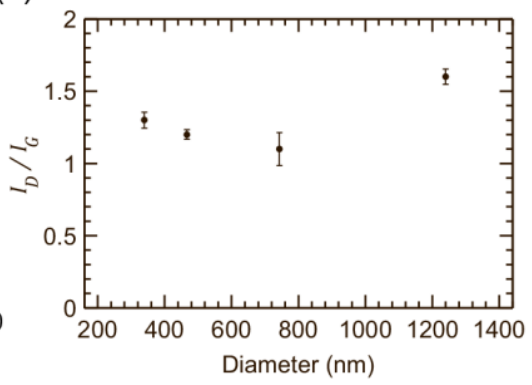


Fig. 3. (a) Maximum current density supported in CNFs of different lengths before electrical breakdown. (b) Raman spectra for CNF of $D=467 \mathrm{~nm}$ and $L=36.7 \mu \mathrm{m}$. (c) $I_{D} / I_{G}$ ratio for fibers of different diameters, the error bars represent the standard error.

\subsection{Downscaling of nanogap size}

In Fig. 4a and Fig. 4b, we show Scanning Electron Microscopy (SEM) micrographs of two CNFs with different lengths, and in Fig. 4c and Fig 4d their respective nanogaps after Joule heating. The nanogap size was measured by SEM and the average values, as well as standard deviations of the forty samples tested were obtained. A comparison between Fig. $4 \mathrm{c}$ and Fig. $4 \mathrm{~d}$ illustrates the reduction of the gap from $G=140 \mathrm{~nm}$ to $\sim 12 \mathrm{~nm}$ as $L$ is scaled down from $39.2 \mu \mathrm{m}$ to $5.0 \mu \mathrm{m}$. These results are summarized in Fig. 4e, where the nanogap size dependence on the length of CNFs is presented, with a clear trend of longer wires producing larger gaps. Curve fitting of the experimental data shows a slightly non-linear dependency of the nanogap size with the length of the wire. Moreover, we found that the ratio between gap size and length, $G / L$, remained below $0.5 \%$ for all samples tested, with a value of $0.24 \%$ for the shortest nanogap produced (Fig. $4 \mathrm{~d}$ ). Following the observed trend, in order to obtain sub-12 nm gaps, CNFs with $L$ shorter than $5 \mu \mathrm{m}$ would be needed. No clear relation was observed between the nanogap size and the diameter of the CNFs, which can be explained by the fact that thin, one-dimensional nanowires have an approximately negligible radial temperature gradient [57]. In such wires, only the axial temperature gradient plays a significant role in the temperature distribution, therefore leading to nanogap sizes dependent on their length only. 

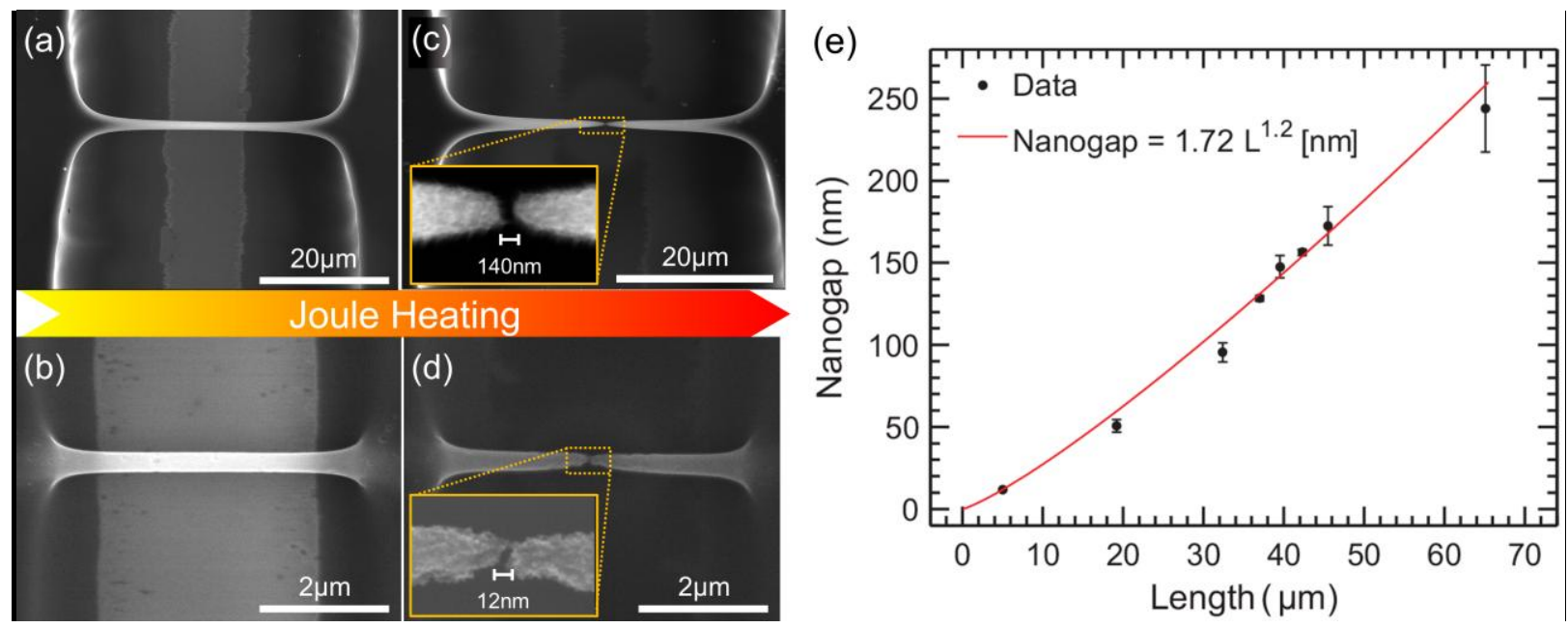

Fig. 4. Nanogaps and their dependence on the downscaling of CNFs. (a,b) SEM micrographs of suspended CNFs prior to electrical breakdown: (a) $L=39.2 \mu \mathrm{m}, D=1180 \mathrm{~nm}$ and (b) $L=5 \mu \mathrm{m}$, $D=289 \mathrm{~nm} .(\mathrm{c}, \mathrm{d})$ Nanogaps produced after Joule heating, with gap size of: (c) $140 \mathrm{~nm}$ and (d) 12 nm. (e) Dependence of gap size on length; each point represents fibers of similar length and error bars represent the standard deviation of the nanogap size. Only one fiber was tested for $L=5 \mu \mathrm{m}$.

\subsection{Atmosphere effects on nanogaps}

In Fig. 5a, a fiber ( $L=65 \mu \mathrm{m}, D=1.06 \mu \mathrm{m})$ broken in air is presented; for comparison, an adjacent CNF of similar dimensions $(L=65 \mu \mathrm{m}, D=1.10 \mu \mathrm{m})$ broken in vacuum is shown in Fig. $5 \mathrm{~b}$. Their very different morphologies suggest that the presence of oxygen and other gases in air leads to different nanogap formation mechanisms. Different magnifications are shown in Fig. 5a and Fig. $5 \mathrm{~b}$, since the CNF broken in vacuum presented a wider gap, likely because a higher voltage than the threshold value was used, leading to uncontrolled thermal runaway of the sample. Previously, it has been reported how multiwalled-CNTs broken in air [20] and CNTs heated in atmospheres with different oxygen concentrations [19] presented larger gaps, compared to samples tested in high vacuum; however, our study is not conclusive enough to support this claim in the case of 
$\mathrm{CNFs}$, and more tests are needed to analyze the effects of partial $\mathrm{O}_{2}$ pressure in the size of the nanogap.

Nonetheless, I-V tests conducted in CNFs under different atmospheres allowed us to study their current carrying capacities qualitatively. Maeda et al. [21] have previously reported on the Joule heating activated desorption of gas molecules on CVD grown fibers, that are responsible in part for charge carrier trapping. This desorption leads to a temporary or permanent reduction in the resistance of the CNF, depending on whether the fiber was electrically biased in air or in vacuum, respectively. The same behavior was observed in our experiments for electromechanically spun CNFs. In Fig. 5c we show the resistance change after four successive bias cycles in air for the fiber in Fig. 5a. As expected, after each heat application, the resistance decreases (due to the increased current), and after cooling down, the next heating-cooling cycle starts at the same resistance value as the previous one. In Fig. $5 \mathrm{~d}$ we show four successive bias cycles for the exact same fiber without breaking it, but operated in vacuum this time. In this case, after each successive sweep, the resistance undergoes a slight permanent reduction. Further, in Fig. 5e it is observed how a CNF broken in air supports only $\sim 35 \%$ of the power supported by one broken in vacuum. These observations are consistent with experiments carried out in CNTs and CNFs, which show that samples electrically stressed in air conditions support lower electrical power before failure [20]. Thus, in order to develop a controllable manufacturing method of nanogap electrodes based on these CNFs, it is important to ensure the stability and purity of the breakdown process by conducting the experiments in vacuum. 

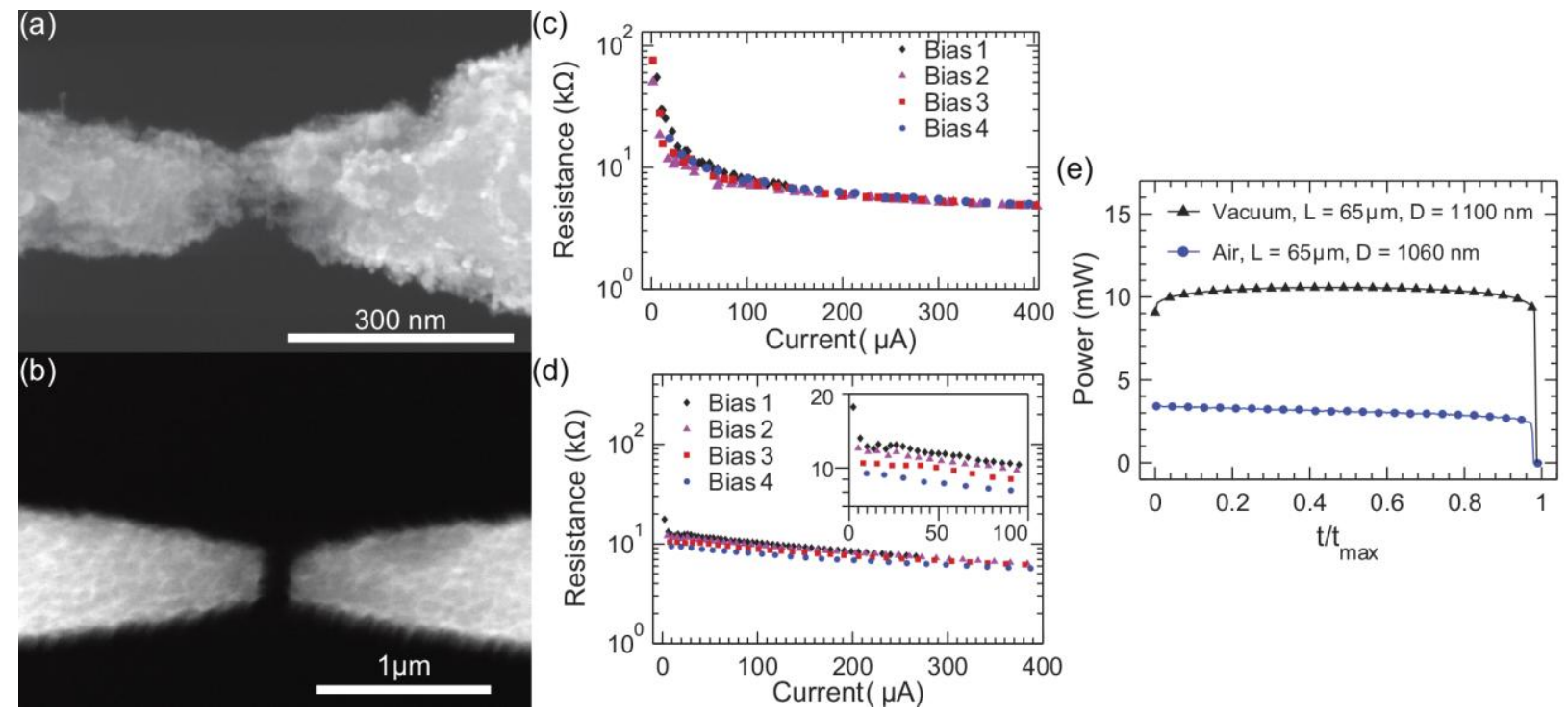

Fig. 5. (a) SEM micrograph of a CNF broken in an air atmosphere ( $L=65 \mu \mathrm{m}$ and $D=1.06 \mu \mathrm{m})$.

(b) SEM micrograph of a CNF broken in vacuum $(L=65 \mu \mathrm{m}$ and $D=1.10 \mu \mathrm{m})$. (c) Resistance change after successive bias cycles applied to the CNF in (a). (d) Resistance change after successive bias cycles in vacuum to the $\mathrm{CNF}$ in (b); inset shows a close-up view evidencing the resistance reduction after each stressing cycle. (e) Comparison of atmosphere effects on the critical power reached in similar CNFs within an array.

\subsection{Simulation of Joule heating temperature}

In order to gain insight into the dependence of Joule heating on fiber geometry, we studied the change in the temperature distribution for different lengths using a three dimensional finite element simulation. The model solved the electric field and current density from the imposed boundary conditions to derive the temperature profile in steady state by treating the electric current as heat source. Two boundary conditions were established for the model: i) the voltage across the carbon electrodes was set to $V_{0}$; ii) the base of the supporting walls, which are in contact with the substrate, remain at room temperature $(293.15 \mathrm{~K})$. Moreover, the model 
considered radiative heat losses, but neglected convective heat transport because samples are considered to be in high vacuum conditions. Five different wire geometries were tested in the simulation consisting of a suspended cylindrical fiber with constant $D=1 \mu \mathrm{m}$ and lengths $L=5$, $10,20,40$ and $65 \mu \mathrm{m}$. Fig. 6 a illustrates the temperature increase in the case $L=40 \mu \mathrm{m}$, where the two ends can be seen to be at significantly lower temperature compared to the central region. This result is in qualitative agreement with the experimental observation of wire breakdown in the middle portion. Furthermore, Fig. $6 \mathrm{~b}$ presents plots of the temperature along the central axis line of the cylindrical wire in Fig. 6a (bounded by the carbon electrodes), as well as for other values of $L$. These plots demonstrate that a large parabolic temperature variation is contained within progressively shorter lengths, with electrodes acting as heat sinks that cool down the ends of the wire.
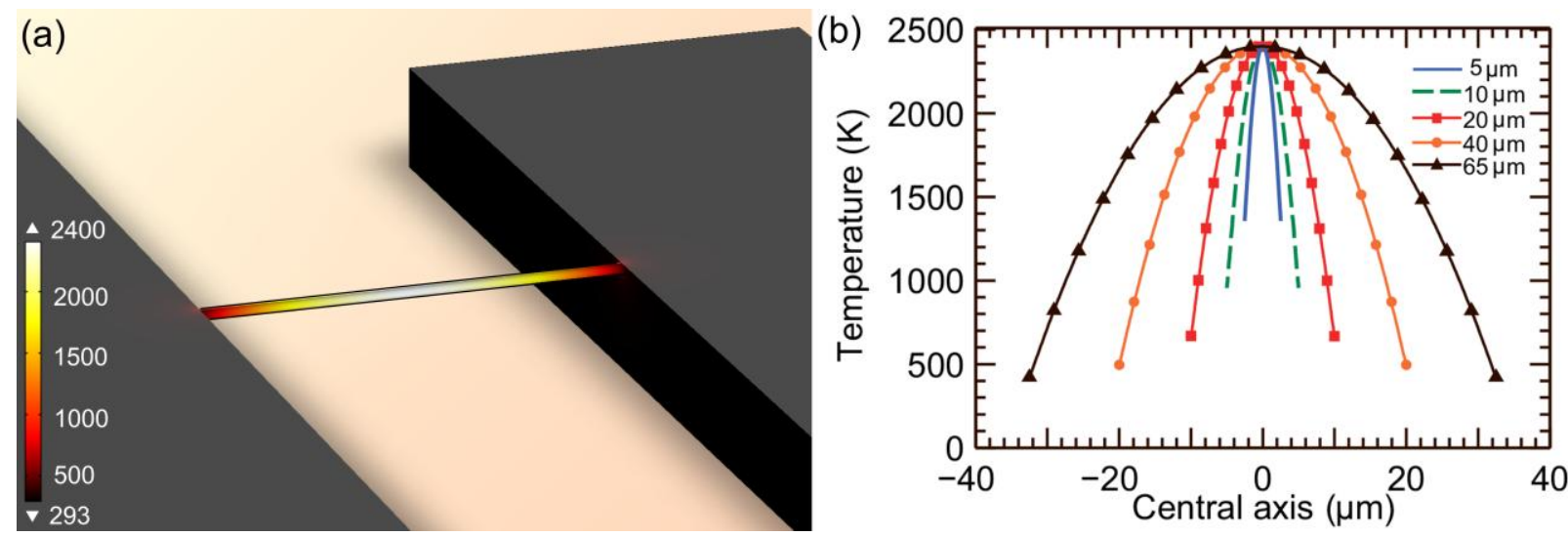

Fig. 6. Simulations of the temperature distribution in carbon fibers due to Joule heating. (a) Colormap plot featuring the temperature profile of a fiber with length $L=40 \mu \mathrm{m}$ and $D=1 \mu \mathrm{m}$. (b) Temperature along the axis of the fiber, for a diameter of $1 \mu \mathrm{m}$ and five different lengths. (A color version of this figure can be viewed online). 
We now turn our discussion to the minimum voltage required to break a CNF. The problem of defining the exact boundary conditions at which a given wire with specific geometry will break is non-trivial. Carbon fibers are expected to withstand a certain critical temperature $T_{\mathrm{m}}$ depending upon the conditions of the atmosphere. In other carbon allotropes, such as multiwalled CNTs, this temperature has been estimated to be around $2400 \mathrm{~K}$ [58], and $3200 \mathrm{~K}$ [20,59] in high vacuum conditions. In the case of the lower bound, the value also corresponds to the sublimation temperature of graphite in vacuum conditions [60]. Because the electrospun CNFs presented in this work are expected to be a mixture of glassy and graphitic carbons $[26,45]$, they are likely less thermodynamically stable than multi-walled CNTs, therefore suggesting that their breaking point lies around $2400 \mathrm{~K}$ in a vacuum. Furthermore, we expect that for a given CNF with specific geometry, there will be a certain minimum threshold voltage $V_{\mathrm{m}}$ that will cause the structure to heat up to maximum temperature $T_{\mathrm{m}}$; in our experiments, this value was in the range between 2 and $6 \mathrm{~V}$. Evidently, any higher bias $V_{0} \geq V_{\mathrm{m}}$ would also break the wire due to excessive Joule heating.

The applied voltage boundary condition, $V_{0}$, was estimated so that wires of the different $L$ would reach the same critical temperature (see Fig. 6b). In the simulation, these values corresponded to $2.372,2.468,2.539,2.606$ and $2.682 \mathrm{~V}$ for fibers with lengths $L=5,10,20,40$ and $65 \mu \mathrm{m}$, respectively. Comparison of these points to the values where nonlinearity starts in IV curves presented in Fig. 2a suggests that the calculated voltages provide a good estimation of the stimulation conditions necessary to begin electrical breakdown. Through this parametric study, length can be directly compared against the temperature gradient that is known to cause electric breakdown in samples of different $L$. From Fig. 6b, it is seen that length has an impact on the temperature gradient, with shorter samples featuring narrower temperature distributions. This 
length dependency becomes evident if we consider that CNFs follow the classical Joule heating heat equation. For a thin, approximately one-dimensional cylindrical wire system with crosssectional area $A$, this equation reads [59]:

$$
A \nabla(\kappa \nabla T)+(I \cdot V) / L=0
$$

where $\kappa$ is the thermal conductivity, $T$ the temperature, $I$ the applied current, $V$ the applied voltage and $L$ the length. It can be shown that the solution to Equation (1) is given by [58]:

$$
T(x)=T_{\mathrm{m}}+\left(T_{0}-T_{\mathrm{m}}\right)\left(\frac{2 x}{L}\right)^{2}
$$

where $T_{0}$ is the temperature at the ends, $T_{\mathrm{m}}$ the maximum temperature reached at the center, given by $T_{\mathrm{m}}=T_{0}+\frac{L}{8 \kappa A}(I \cdot V)$, and $x \in[-L / 2, L / 2]$. Notice that in Equation (2), the dependence of the thermal and electrical conductivities with $T$ has been neglected in the lowest order approximation. Next, we consider the case in which fibers have the same cross-sectional area $A$ and break at the same critical temperature $T_{\mathrm{m}}$. In this scenario, Equation (2) evinces that the temperature gradient is directly related to the geometrical features of the wire. In shorter CNFs, the second term of Equation (2) will increase in value, resulting in a steeper temperature gradient solution, which corresponds to a more localized high temperature region in the middle of the CNF and thus smaller nanogaps. These results, along with the computed temperature distributions (Fig. 6b) are consistent with the experimental evidence of nanogap scaling with length illustrated in Fig. 4e.

\section{Conclusions}

We have demonstrated a simple and inexpensive manufacturing technique for the fabrication of single suspended CNFs, that can be used to create $\sim 12 \mathrm{~nm}$ nanogaps through Joule heating. The merging of EMS and UV-photolithography allowed us to control the length, positioning and 
orientation of freestanding CNFs, as well as the fabrication of devices with multiple wires. Furthermore, CNFs were monolithically integrated with carbon electrodes, which obviated the use of complex postbonding steps. This freedom of design enabled the electrical testing of different $\mathrm{CNF}$ geometries under vacuum and ambient conditions. We found that the nanogap dimensions and the maximum current density in CNFs $\left(\sim 10^{5} \mathrm{~A} / \mathrm{cm}^{2}\right)$ are length dependent. Moreover, fibers tested in ambient conditions experienced a $65 \%$ reduction in supported power when compared to samples tested in vacuum with similar geometries.

The length dependence of nanogaps was studied through computational simulation, which suggested that the temperature distribution of Joule heated wires is parabolic, with increasingly steeper temperature profiles for shorter samples. Following that trend, to obtain smaller gaps, as required for most molecular electronics applications, CNFs shorter than $5 \mu \mathrm{m}$ are needed. The challenge in obtaining shorter fibers with the proposed methodology lies in the limitations of the conventional UV-photolithography, where the fabrication of supporting SU-8 walls becomes more complex and delicate for smaller features. Therefore, optimization and improvement of the current photolithography set-up is required to produce short enough CNFs.

\section{Acknowledgments}

This work was financially supported by NSF grant no. 1449397, CONACYT grant no. CB2014-1-241458, CONACYT postdoctoral fellowship no. 232499, and the Sensors and Devices Group of Tecnologico de Monterrey. SEM work was performed at the UC Irvine Materials Research Institute (IMRI), using instrumentation funded in part by the National Science Foundation Center for Chemistry at the Space-Time Limit (CHE-082913). 


\section{References}

[1] T. Li, W. Hu, D. Zhu, Nanogap Electrodes, Adv. Mater. 22 (2) (2010) 286-300. doi:10.1002/adma.200900864.

[2] A. Kuzyk, Dielectrophoresis at the nanoscale, Electrophoresis, 32 (17) (2011) 23072313. doi:10.1002/elps.201100038.

[3] X. Chen, Z. Guo, G. M. Yang, J. Li, M. Q. Li, J. H. Liu, et al., Electrical nanogap devices for biosensing, Mater. Today, 13 (11) (2010), 28-41. doi:10.1016/S1369-7021(10)702017.

[4] A. Cui, H. Dong, W. Hu, Nanogap Electrodes towards Solid State Single-Molecule Transistors, Small, 11 (46) (2015), 6115-6141. doi:10.1002/smll.201501283.

[5] A. Fursina, S. Lee, R. G. S. Sofin, I. V. Shvets, D. Natelson, Nanogaps with very large aspect ratios for electrical measurements, Appl. Phys. Lett., 92 (11) (2008), 113102. doi:10.1063/1.2895644.

[6] S. Roy, H. Vedala, A. D. Roy, D.-H. Kim, M. Doud, K. Mathee, et al., Direct electrical measurements on single-molecule genomic DNA using single-walled carbon nanotubes, Nano Lett., 8 (1) (2008), 26-30. doi: 10.1021/n10716451.

[7] D. Cheon, S. Kumar, G. H. Kim, Assembly of gold nanoparticles of different diameters between nanogap electrodes, Appl. Phys. Lett., 96 (1) (2010), 013101. doi:10.1063/1.3280859.

[8] A. Vijayaraghavan, S. Blatt, D. Weissenberger, M. Oron-Carl, F. Hennrich, D. Gerthsen, et al., Ultra-large-scale directed assembly of single-walled carbon nanotube devices, Nano Lett., 7 (6) (2007), 1556-60. doi:10.1021/nl0703727.

[9] T. Nagase, K. Gamo, T. Kubota, S. Mashiko, Direct fabrication of nano-gap electrodes by focused ion beam etching, Thin Solid Films, 499 (1-2) (2006), 279-284, Mar. 2006. doi:10.1016/j.tsf.2005.07.031.

[10] W. Lee, K. Kim, W. Jeong, L. A. Zotti, F. Pauly, J. C. Cuevas, et al., Heat dissipation in atomic-scale junctions, Nature, 498 (7453) (2013), 209-212. doi:10.1038/nature12183.

[11] D.R. Strachan, D.E. Smith, D.E. Johnston, T.H. Park, M.J. Therien, D.A. Bonnell, et al., Controlled fabrication of nanogaps in ambient environment for molecular electronics, Appl. Phys. Lett. 86 (4) (2005) 43109. doi:10.1063/1.1857095.

[12] M. L. Perrin, C. J. O. Verzijl, C. A. Martin, A. J. Shaikh, R. Eelkema, J. H. Van Esch, et al., Large tunable image-charge effects in single-molecule junctions, Nat. Nanotechnol., 8 (4) (2013), 282-287. doi:10.1038/nnano.2013.26.

[13] D. J. Beesley, J. Semple, L. Krishnan Jagadamma, A. Amassian, M. A McLachlan, T. D. Anthopoulos, et al., Sub-15-nm patterning of asymmetric metal electrodes and devices by adhesion lithography., Nat. Commun., 5 (2014), 3933. doi:10.1038/ncomms4933.

[14] C. Thiele, M. Engel, F. Hennrich, M. M. Kappes, K. P. Johnsen, C. G. Frase, et al., Controlled fabrication of single-walled carbon nanotube electrodes by electron-beaminduced oxidation, Appl. Phys. Lett., 99 (17) (2011), 173105. doi:10.1063/1.3656736.

[15] J. H. Kim, H. Moon, S. Yoo, Y. K. Choi, Nanogap electrode fabrication for a nanoscale device by volume-expanding electrochemical synthesis, Small, 7 (15) (2011), 22102216. doi:10.1002/smll.201002103. 
[16] S. Kubatkin, A. Danilov, M. Hjort, J. Cornil, J.-L. Brédas, N. Stuhr-Hansen, et al., Single-electron transistor of a single organic molecule with access to several redox states, Nature, 425 (6959) (2003), 698-701. doi:10.1002/smll.201002103.

[17] H. Zhang, S. W. Chung, and C. A. Mirkin, Fabrication of sub-50-nm solid-state nanostructures on the basis of dip-pen nanolithography, Nano Lett., 3 (1) (2003), 43-45. doi: $10.1021 / \mathrm{nl} 0258473$.

[18] G. A. Kalkman, Y. Zhang, E. Monachino, K. Mathwig, M. H. E. Kamminga, P. Pourhossein, et al., Bisecting Microfluidic Channels with Metallic Nanowires Fabricated by Nanoskiving, ACS Nano, 10 (2) (2016), 2852-2859. doi:10.1021/acsnano.5b07996.

[19] C. W. Marquardt, S. Grunder, A. Błaszczyk, S. Dehm, F. Hennrich, H. v. Löhneysen, et al., Electroluminescence from a single nanotube-molecule-nanotube junction, Nat. Nanotechnol., 5 (12) (2010), 863-867. doi:10.1038/nnano.2010.230.

[20] P.G. Collins, M. Hersam, M. Arnold, R. Martel, P. Avouris, Current Saturation and Electrical Breakdown in Multiwalled Carbon Nanotubes, Phys. Rev. Lett. 86 (14) (2001) 3128-3131. doi:10.1103/PhysRevLett.86.3128.

[21] S. Maeda, P. Wilhite, N. Kanzaki, T. Yamada, C.Y. Yang, Change in carbon nanofiber resistance from ambient to vacuum, AIP Adv. 1 (2) (2011) 22102. doi:10.1063/1.3582812.

[22] M.A. Reed, C. Zhou, C.J. Muller, T.P. Burgin, J.M. Tour, Conductance of a Molecular Junction, Science. 278 (5336) (1997) 252-254. doi:10.1126/science.278.5336.252.

[23] X. Guo, J.P. Small, J.E. Klare, Y. Wang, M.S. Purewal, I.W. Tam, et al., Covalently bridging gaps in single-walled carbon nanotubes with conducting molecules., Science. 311 (5759) (2006) 356-9. doi:10.1126/science.1120986.

[24] X. Guo, A.A. Gorodetsky, J. Hone, J.K. Barton, C. Nuckolls, Conductivity of a single DNA duplex bridging a carbon nanotube gap., Nat. Nanotechnol. 3 (163) (2008) 163-7. doi:10.1038/nnano.2008.4.

[25] P.G. Collins, M.S. Arnold, P. Avouris, Engineering Carbon Nanotubes and Nanotube Circuits Using Electrical Breakdown, Science. 292 (5517) (2001) 706-709. doi:10.1126/science.1058782.

[26] S. Sharma, A. Sharma, Y.-K. Cho, M. Madou, Increased Graphitization in Electrospun Single Suspended Carbon Nanowires Integrated with Carbon-MEMS and Carbon-NEMS Platforms, ACS Appl. Mater. Interfaces. 4 (1) (2012) 34-39. doi:10.1021/am2014376.

[27] J.H. Han, K. Song, S. Radhakrishnan, S.H. Oh, C.H. Lee, A suspended nanogap formed by field-induced atomically sharp tips, Appl. Phys. Lett. 101 (18) (2012) 183106. doi:10.1063/1.4764562.

[28] C. H. Jin, J. Y. Wang, Q. Chen, L. M. Peng, In situ fabrication and graphitization of amorphous carbon nanowires and their electrical properties, J. Phys. Chem. B, 110 (11) (2006), 5423-5428. doi:10.1021/jp057240r.

[29] S.B. Lee, K.B.K. Teo, L.A.W. Robinson, A.S. Teh, M. Chhowalla, D.G. Hasko, et al., Characteristics of multiwalled carbon nanotube nanobridges fabricated by poly(methylmethacrylate) suspended dispersion, J. Vac. Sci. Technol. B. 20 (6) (2002) 2773-2776. doi:10.1116/1.1520569.

[30] C. Wang, M. Madou, From MEMS to NEMS with carbon, Biosens. Bioelectron., 20 (10) (2005), 2181-2187. doi:10.1016/j.bios.2004.09.034. 
[31] M. Suzuki, Y. Ominami, Q. Ngo, C.Y. Yang, A.M. Cassell, J. Li, Current-induced breakdown of carbon nanofibers, J. Appl. Phys. 101 (11) (2007) 114307. doi:10.1063/1.2743086.

[32] H. Kitsuki, T. Yamada, D. Fabris, J.R. Jameson, P. Wilhite, M. Suzuki, et al., Length dependence of current-induced breakdown in carbon nanofiber interconnects, Appl. Phys. Lett. 92 (17) (2008) 173110. doi:10.1063/1.2918839.

[33] L. A. Pesin, Review: Structure and properties of glass-like carbon, J. Mater. Sci. 37 (1) (2002). 1-28. doi:10.1023/A:1013100920130.

[34] B.Y. Park, L. Taherabadi, C. Wang, J. Zoval, M.J. Madou, Electrical Properties and Shrinkage of Carbonized Photoresist Films and the Implications for Carbon Microelectromechanical Systems Devices in Conductive Media. J. Electrochem. Soc. 152 (12) (20015), J136-J143. doi:10.1149/1.2116707.

[35] C.S. Sharma, H. Katepalli, A. Sharma, M. Madou, Fabrication and electrical conductivity of suspended carbon nanofiber arrays, Carbon. 49 (5) (2011) 1727-1732. doi:10.1016/j.carbon.2010.12.058.

[36] S. Sharma, A. Sharma, Y.-K. Cho, M. Madou, Increased Graphitization in Electrospun Single Suspended Carbon Nanowires Integrated with Carbon-MEMS and Carbon-NEMS Platforms, ACS Appl. Mater. Interfaces. 4 (1) (2012) 34-39. doi:10.1021/am2014376.

[37] A. A. Balandin, Thermal properties of graphene and nanostructured carbon materials, Nat. Mater., 10 (8) (2011), 569-581. doi:10.1038/nmat3064.

[38] B.A. Samuel, R. Rajagopalan, H.C. Foley, M.A. Haque, Temperature effects on electrical transport in semiconducting nanoporous carbon nanowires, Nanotechnology. 19 (17) (2008) 275702. doi:10.1088/0957-4484/19/27/275702.

[39] C. M. Lentz, B. A. Samuel, H. C. Foley, M. A. Haque, Synthesis and Characterization of Glassy Carbon Nanowires, J. Nanomater., 2011 (2010), 1-8. doi:10.1155/2011/129298.

[40] A. Tibrewala, E. Peiner, R. Bandorf, S. Biehl, H. Lüthje, Transport and optical properties of amorphous carbon and hydrogenated amorphous carbon films, Appl. Surf. Sci., 252 (15) (2006), 5387-5390. doi:10.1016/j.apsusc.2005.12.046.

[41] D.H. Reneker, A.L. Yarin, H. Fong, S. Koombhongse, Bending instability of electrically charged liquid jets of polymer solutions in electrospinning, J. Appl. Phys. 87 (9) (2000) 4531-4547. doi:10.1063/1.373532.

[42] J. Doshi, D.H. Reneker, Electrospinning process and applications of electrospun fibers, J. Electrost. 35 (2-3) (1995) 151-160. doi:10.1016/0304-3886(95)00041-8.

[43] D. Sun, C. Chang, S. Li, L. Lin, Near-Field Electrospinning, Nano Lett. 6 (4) (2006) 839-842. doi:10.1021/n10602701.

[44] G.S. Bisht, G. Canton, A. Mirsepassi, L. Kulinsky, S. Oh, D. Dunn-Rankin, et al., Controlled continuous patterning of polymeric nanofibers on three-dimensional substrates using low-voltage near-field electrospinning, Nano Lett. 11 (4) (2011) 1831-1837. doi: $10.1021 / \mathrm{nl} 2006164$.

[45] G. Canton, T. Do, L. Kulinsky, M. Madou, Improved conductivity of suspended carbon fibers through integration of C-MEMS and Electro-Mechanical Spinning technologies, Carbon. 71 (2014) 338-342. doi: 10.1016/j.carbon.2014.01.009.

[46] G. Canton, Development of Electro-Mechanical Spinning for Controlled Deposition of Carbon Nanofibers, University of California Irvine, 2014. 
[47] J.Y. Huang, S. Chen, Z.F. Ren, G. Chen, M.S. Dresselhaus, Real-Time Observation of Tubule Formation from Amorphous Carbon Nanowires under High-Bias Joule Heating, Nano Lett. 6 (8) (2006) 1699-1705. doi:10.1021/n10609910.

[48] T. Saito, T. Yamada, D. Fabris, H. Kitsuki, P. Wilhite, M. Suzuki, et al., Improved contact for thermal and electrical transport in carbon nanofiber interconnects, Appl. Phys. Lett. 93 (10) (2008) 102108. doi:10.1063/1.2979710.

[49] O.J.A. Schueller, S.T. Brittain, C. Marzolin, G.M. Whitesides, Fabrication and characterization of glassy carbon MEMS, Chem. Mater. 9 (6) (1999) 1399-1406. doi: $10.1021 / \mathrm{cm} 960639 \mathrm{v}$.

[50] Y.S. Virgil'ev, I.G. Lebedev, Effect of neutron irradiation on properties of glassy carbon, Inorg. Mater. 38 (7) (2002) 668-673. doi:10.1023/A:1016232223288.

[51] C. Yu, S. Saha, J. Zhou, L. Shi, A.M. Cassell, B.A. Cruden, et al., Thermal Contact Resistance and Thermal Conductivity of a Carbon Nanofiber, J. Heat Transf. 128 (3) (2006) 234-239. doi:10.1115/1.2150833.

[52] M. Balat-Pichelin, J.F. Robert, J.L. Sans, Emissivity measurements on carbon-carbon composites at high temperature under high vacuum, Appl. Surf. Sci. 253 (2) (2006) 778783. doi:10.1016/j.apsusc.2006.01.007.

[53] Y. Lim, J. Heo, M. Madou, H. Shin, Monolithic carbon structures including suspended single nanowires and nanomeshes as a sensor platform, Nanoscale Res. Lett. 8 (1) (2013) 1-9. doi: 10.1149/06107.0025ecst.

[54] Z. Wang, Z. Lu, Y. Huang, R. Xue, X. Huang, L. Chen, Characterizations of crystalline structure and electrical properties of pyrolyzed polyfurfuryl alcohol, J. Appl. Phys. 82 (11) (1997). doi: 10.1063/1.366434.

[55] A. Barreiro, F. Börrnert, M.H. Rümmeli, B. Büchner, L.M.K. Vandersypen, Graphene at High Bias: Cracking, Layer by Layer Sublimation, and Fusing, Nano Lett. 12 (4) (2012) 1873-1878. doi:10.1021/nl204236u.

[56] J.Y. Huang, F. Ding, B.I. Yakobson, P. Lu, L. Qi, J. Li, In situ observation of graphene sublimation and multi-layer edge reconstructions, Proc. Natl. Acad. Sci. 106 (25) (2009) 10103-10108. doi:10.1073/pnas.0905193106.

[57] F. Ĺonard, Reduced Joule heating in nanowires, Appl. Phys. Lett. 98 (10) (2011) 20092012. doi:10.1063/1.3561772.

[58] S.B. Singer, M. Mecklenburg, E.R. White, B.C. Regan, Single-color pyrometry of individual incandescent multiwalled carbon nanotubes, Phys. Rev. B - Condens. Matter Mater. Phys. 84 (19) (2011) 1-8. doi:10.1103/PhysRevB.84.195468.

[59] G.E. Begtrup, K.G. Ray, B.M. Kessler, T.D. Yuzvinsky, H. Garcia, A. Zettl, Probing Nanoscale Solids at Thermal Extremes, Phys. Rev. Lett. 99 (15) (2007) 155901. doi:10.1103/PhysRevLett.99.155901.

[60] F.P. Bundy, Pressure-temperature phase diagram of elemental carbon, Phys. Stat. Mech. Its Appl. 156 (1) (1989) 169-178. doi:10.1016/0378-4371(89)90115-5. 

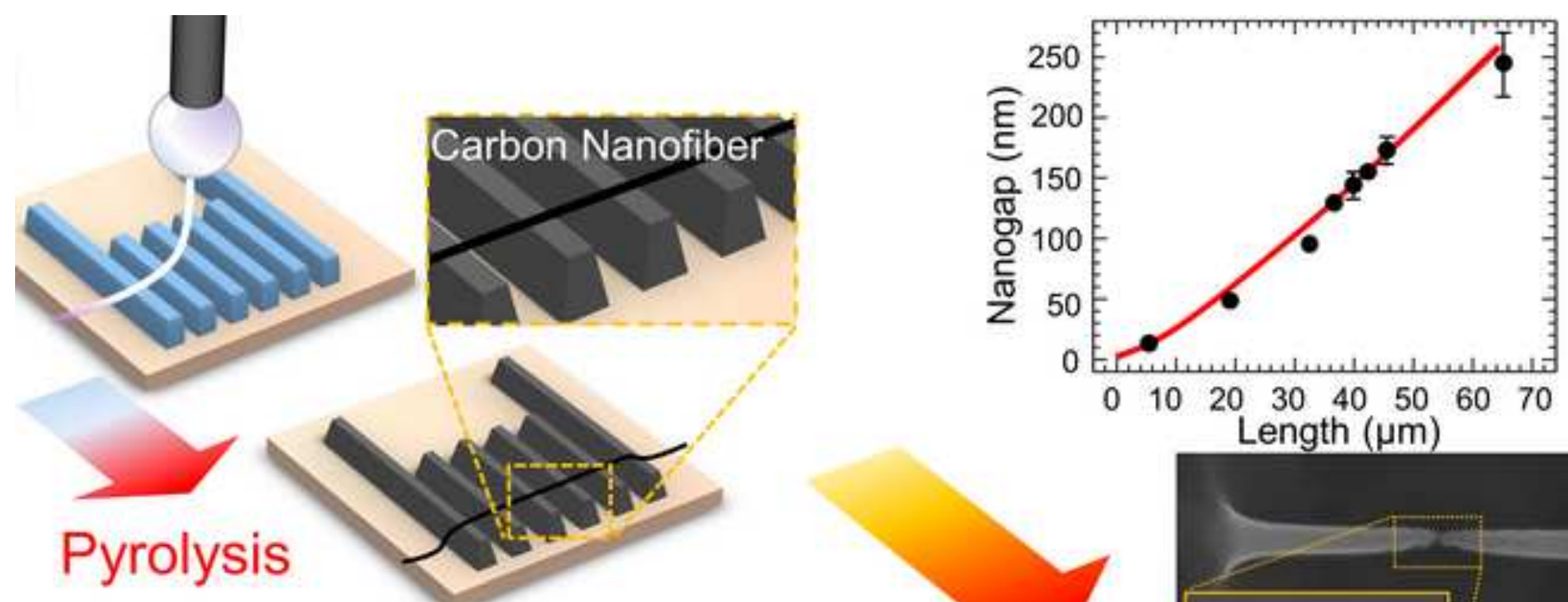

Joule Heating

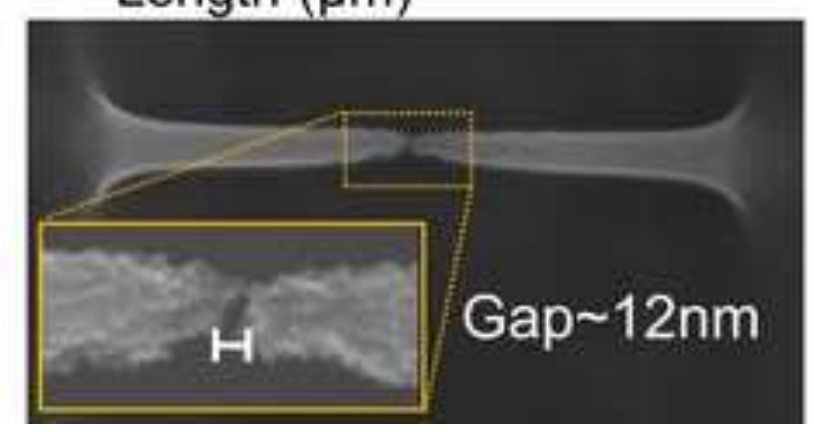

